\title{
STUDY ON THE VARIATION OF SURFACE WATER BASED ON LANDSAT IMAGERY IN GUILIN
}

\author{
Xianjian Lu ${ }^{1}$, ChenLong Wu ${ }^{1}$, Hongbo Yan ${ }^{1, *}$, YuHui Huang ${ }^{1}$, Le Luo ${ }^{1}$, Bin Zhou ${ }^{1}$ \\ ${ }^{1}$ Guilin University of Technology, College of Geomatics and Geoinformation, 541006, Guilin
}

KEY WORDS: Surface Water; Remote Sensing Monitoring; Image Processing; Distribution Changes

\begin{abstract}
:
Using remote sensing technology to study the distribution and change of surface water is an important application of remote sensing technology. It is also an important means of water resources protection. This paper takes the Lijiang River Basin in Guilin, Guangxi as the research object. Landsat satellite images were used as the data source. After classifying the images, the surface water distribution area of Lijiang River Basin in Guilin City was extracted, and the area change and its spatial distribution are compared. On this basis, combined with the actual situation in Guilin, the paper analyzes the causes of surface water distribution change in Lijiang river basin from two aspects of natural factors and human factors.
\end{abstract}

\section{INTRODUCTION}

Located in the northeastern part of Guangxi, Guilin is a worldfamous tourist city. Guilin's "Two Rivers and Four Lakes" has become the golden waterway of world tourism with its natural beauty, and it is also the source of life for the city to survive and develop (Zhu, 2003). At the same time, surface water is also the main source of human life and production, so it is very important to do a good job in monitoring the distribution of surface water. However, due to the different forms of surface water and the complex and varied river areas and the incompleteness of traditional monitoring systems, it is difficult to obtain ground information directly through traditional means. Based on the application of remote sensing technology in surface water distribution monitoring, this paper analyzes the changes of surface water distribution in Guilin and provides scientific basis for the protection of water resources in Guilin.

Remote sensing change monitoring technology began in 1960s, which is a comprehensive application of digital image processing method, computer vision technology, artificial intelligence and pattern recognition theory. With the development of remote sensing technology and the improvement of surface water monitoring technology, remote sensing has achieved certain application results in surface water hydrological monitoring.

Foreign scholars Frappart used the water surface area extracted by multi-spectral remote sensing data and the water level data monitored by ground hydrological stations to estimate the monthly relative water storage of Me Kong river. In the relevant research fields in China, Qi Shuhua et al. used Landsat data to extract Poyang Lake water body. Boundary, and by means of superposition, classification and other methods to generate images with a resolution of $30 \mathrm{~m}$, the water depth distribution and water storage of Poyang Lake (Wang, 2007).

In 2006, Huang Weihua used MODIS real-time satellite data to carry out long-term remote sensing dynamic monitoring of the surface water area of reservoirs, ponds and dams in Guangxi, effectively and objectively reflecting the changes in local drought conditions, which made up for other droughts. The shortcomings of remote sensing monitoring methods are insufficient. The practice has proved that remote sensing dynamic monitoring of surface water bodies is an effective method for surface water distribution monitoring( $\mathrm{Li}$ et al., 2008).

\section{DATA RESOURCES AND STUDY AREA}

\subsection{Technical Methods For Remote Sensing Monitoring Of Surface Water}

In recent years, with the continuous development of remote sensing technology, various high-precision satellite image data products have been widely used, and using satellite data to extract water information on the land surface has become an important method for water resources investigation, flood disaster assessment and ecological environment monitoring, etc. In remote sensing monitoring of surface water, remote sensing images are used for water monitoring(Fan et al., 2010). Specific methods are as follows:

1. Single-band or multi-band threshold method is mainly used to select one or more bands that can better reflect the boundary between water body and other ground objects and determine the threshold value according to the difference of gray value between different ground objects in remote sensing images, so as to extract the water body.

2. NDVI method is to extract water bodies from MODIS images by using normalized vegetation index composed of surface reflectance in band 1 and band 2 of MODIS data.

3. Spectral correlation method: according to the difference and change rule of spectral curves of water, vegetation, city, soil and other background features, the relationship between different wavebands is expressed by logical discriminant, and then the water is extracted. Compared with NDVI method, this method can extract water pixel more quickly and accurately. 4. The water index method mainly selects multiple bands closely related to water identification according to the spectral characteristics of water, and constructs the water index by using

\footnotetext{
* Corresponding author
} 
the mapping relationship between water and remote sensing spectral values, so as to achieve the extraction of water information.

5. Water monitoring system with spatial information technology to support, database technology as the foundation platform, factors such as extraction and water body is closely related to the spatial and temporal distribution of multi-source information, establish water monitoring database and water monitoring system, realize the water resource management informatization, make the development and utilization of water resources, water conservancy project management based on timely, accurate and scientific information, better service for the sustainable development of water (Liu, 2008).

MODIS data obtained from remote sensing images can be used to extract water information quickly and effectively. It is suitable for the present situation and change of water body in large area. Combined with the appeal method, a variety of water indexes are comprehensively used, and the reflection characteristics of the actual ground objects are combined, which will help to eliminate the interference of various non-water pixels in the image and extract the real water information. The deficiency of MODIS data is that the spatial resolution of each band is only $250 \mathrm{~m}$, which makes it difficult to identify small and medium-sized rivers and ponds in remote sensing images.

\subsection{Overview of the Research Area}

Guilin City was located in the northeastern part of Guangxi Zhuang Autonomous Region, which belongs to the transitional zone between the western section of Nanling Mountain and the Guizhou Plateau. It is mainly composed of three major landforms: hilly, mountain and karst basins, and the three terrains cover an area of 2,129 square kilometers, accounting for 76.6 percent, platform area accounts for $23.4 \%$. The location of Guilin is shown in Figure 3-1.

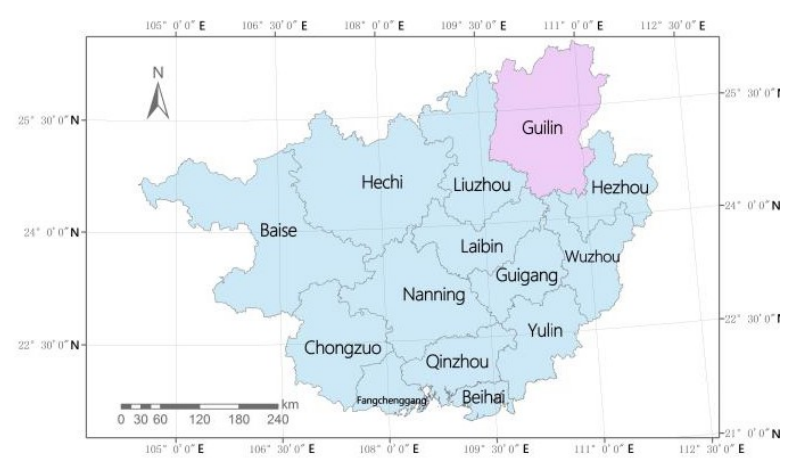

Figure 2-1 geographical location of Guilin city

The downtown area of Guilin is located in the central part of Guilin city, on the Lijiang river valley basin, and distributed in a ribbon on the upper Banks of the Lijiang river in the northsouth direction. It has jurisdiction over 12 counties and the urban area of Guilin, with a total area of 27,809 square kilometers.

Guilin is also the birthplace of Xiangjiang river and Lijiang river. There are 10 rivers with water collecting area of more than 1000 square kilometers in the territory, which are divided into the Yangtze River Basin and the Pearl River Basin, among which the exit rivers are mainly the Xiangjiang river in the Yangtze River Basin, the Zijiang river and the Guijiang river in the Pearl River Basin (called Li river in the upstream), the Luoqing river and Xunjiang river.

Guilin is located at a low latitude, with a warm and humid climate, and belongs to the mid-subtropical monsoon climate zone. There is no severe cold and heat throughout the year. The annual average temperature is between 17 and $20^{\circ} \mathrm{C}$. The temperature increases from north to south. The highest temperature is $39.4{ }^{\circ} \mathrm{C}$ and the lowest temperature is $-5.8{ }^{\circ} \mathrm{C}$. The rain is abundant, and the annual rainfall in most areas is above $1700 \mathrm{~mm}$, and the annual average evaporation is about $1500 \mathrm{~mm}$. The rainy season is generally from March to August, and the maximum rainfall for four consecutive months occurs from April to July (Zhao, 2003). The spatial distribution of rainfall is uneven, generally more in the north than in the south, and the mountainous areas are higher than the valleys and plains The interannual variation of rainfall is related to the strength of the monsoon activity.

\subsection{The Data Source}

In this study, TM remote sensing images from August to December 2010, September to October 2013 and February to April 2016 were selected as image data sources. From the perspective of spatial resolution, the image is relatively low, which makes it difficult to extract small ground objects in the earth class. However, the image has more wavebands and higher spectral resolution, which is conducive to the identification and extraction of ground objects in narrow spectrum (Shafian S et al., 2015).

ENVI5.2 and ENVI5.2 Classic were used for image processing in this study.TM images of Landsat 5 were used in the 2010 images, and Landsat 8 images were used in the 2016 images.

\section{DATA PROCESSING}

\subsection{Image Preprocessing and Parameter Settings}

The process of image preprocessing includes image correction, cutting and splicing.

1) Image Correction

In this study, only the distribution area of surface water and water in Guilin City was extracted for analysis. The geometric correction did not have much influence on the extraction of water. Therefore, the images used in the study were only subjected to radiation correction and atmospheric correction.

The images selected in this study are multi-spectral images. In the radiation correction, the calibration type is Radiance, the storage order is BIL, the data type is Float, and the radiation rate data unit adjustment coefficient is 0.1 , which can automatically perform radiation correction. The spectral curve values of radiometric images are mainly concentrated in the range of $0 \sim 10$. Next, perform atmospheric correction, set a single conversion factor to 1 , and select the sensor type. The 2010 image selects the Landsat5 TM sensor, and the 2013 and 2016 images select the Landsat8 OLI sensor, According to the imaging time of the image, select the parameter and set the K-T inversion mode to Over-Land Retrieval standard (600:2100) to automatically select the corresponding band. High setting can output atmospheric correction result by default. The radiationcorrected and atmospheric-corrected images remove certain measurement errors and result in sharper images.

2) Image Cropping 
The study area is Guilin City, so it is cut according to the vector map data of the municipal administrative divisions of Guilin City, open the administrative boundaries, in the study area in ENVI guilin vector graph as a cutting edge, after correction of the remote sensing image cropping, concrete process is the vector diagram and multiplication by after correction of remote sensing image,the spectrum value within the municipal administrative scope is multiplied by 1 to be retained; The spectral value outside the administrative scope of Guilin city is multiplied by 0 to cancel.According to the calculated results, the cropped image data of the study area can be obtained (Wang, 2003).

3) Image Mosaic

In this study, the four images that were cropped were inlaid into the entire municipal administrative area of Guilin.

In this article, the image mosaic is embedded using the Seamless Mosaic tool in ENVI5.2. In this paper, we use this tool to achieve more fine control of the mosaic of images, and 3.2 Image Classification After Processing

The supervised classification method of computer classification based on spectrum is used in this paper. Supervising classification is the process of using the sample pixels of the to obtain images with more uniform colors and softer edges. Taking the 2016 Guilin image map for cropping and inlay as an example, as shown in figure $3-1$.

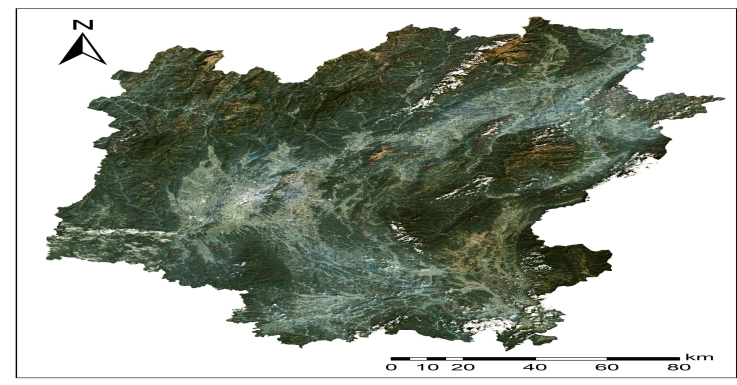

Figure 3-1 image map of Guilin City in 2016

confirmed category to identify the pixels of other unknown categories. Monitoring classification of remote sensing images generally includes the following 6 steps (Chen et al., 2003), as shown in figure 3-2.

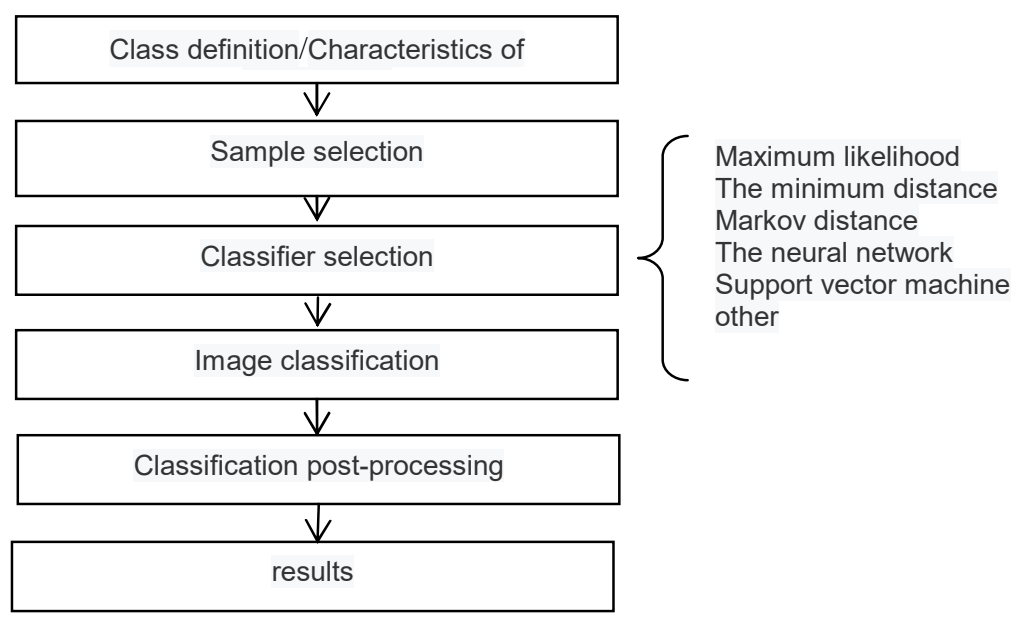

Figure 3-2 supervised classification steps

In this study, the image was first classified, and water information was extracted according to the research needs. Through visual interpretation and feature judgment to identify water features; Next, a certain number of samples are selected for water information, and objects outside water are classified as others. The classifier is selected according to the complexity and accuracy requirements of classification. Using the selected classification samples and classifiers for image classification; Finally, the classification results are evaluated to determine the accuracy and reliability of classification (Bai et al., 1997).

\section{SPATIAL DISTRIBUTION OF SURFACE WATER IN GUILIN}

\subsection{Distribution Pattern Of Surface Water In Guilin}

Through the above supervision classification method can be extracted from the distribution of surface water in guilin. As

\subsection{Post-classification Treatment}

The results obtained after supervised classification are generally preliminary results, which are difficult to achieve the final application purpose. In this study, the method of cluster processing is adopted in the post-classification processing of images to remove small spots from the images, so as to make the images smoother, better picture effect and stronger overall, which lays a good foundation for the following image classification statistics.

shown in figure 4-1 (a) for the surface profile in 2010, guilin, figure 4-1 (b) for the surface profile in 2013, guilin, figure 4-1(c) for the surface profile in 2016, guilin, figure 4-1 (d) is displayed on the image to extract the surface profile of guilin in 2013, figure 4-1 (e) for enlarged lijiang river interchange of surface water and tea river extract: 


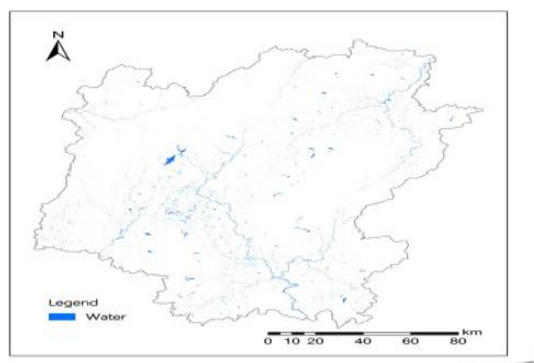

(a)

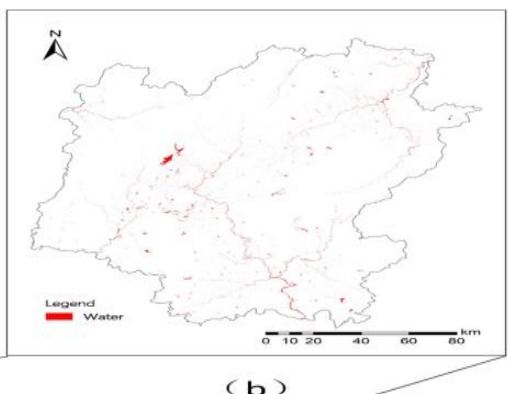

(b)

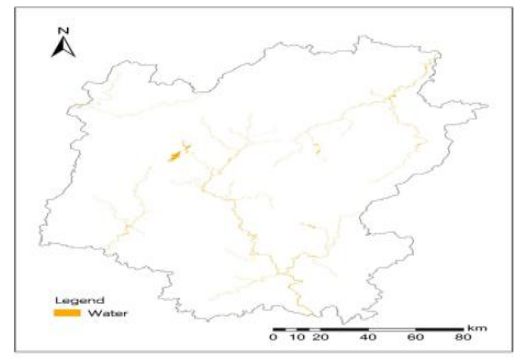

(c)

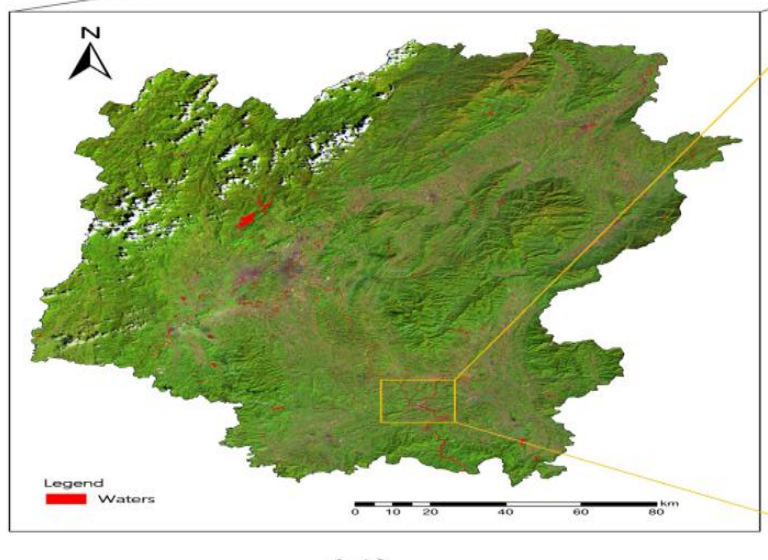

Figure 4-1 surface water distribution map of Guilin

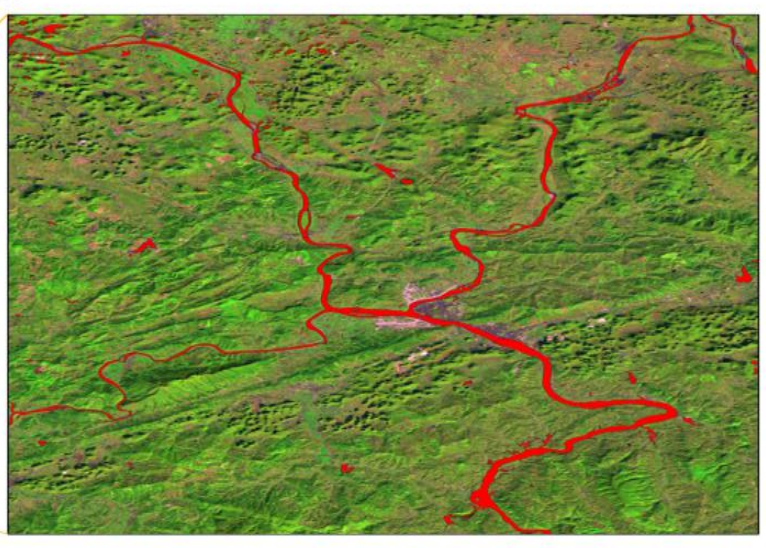

(e)

The main content of this paper is the change of surface water distribution in Guilin. The classification statistics is based on the classification results to calculate the statistical information of the source classification image. After the classification statistics, the statistical results of the surface water distribution in Guilin can be obtained. Statistical results of surface water distribution in Guilin City at three different periods in Table 4-1, Table 4-2 and Table 4-3.

\begin{tabular}{|c|c|c|}
\hline category & As the elements & The percentage \\
\hline unclassified & 0 & 0 \\
The water & 278291 & 0.563449 \\
other & 49112350 & 99.436551 \\
\hline
\end{tabular}

Table 4-1 Statistical results of surface water distribution in Guilin in 2010

\begin{tabular}{|c|c|c|}
\hline category & As the elements & The percentage \\
\hline unclassified & 0 & 0 \\
The water & 288876 & 0.584880 \\
other & 49101765 & 99.415120 \\
\hline
\end{tabular}

Table 4-2 Statistical results of surface water distribution in Guilin in 2013

\begin{tabular}{|c|c|c|}
\hline category & As the elements & The percentage \\
\hline unclassified & 0 & 0 \\
The water & 308133 & 0.623869 \\
other & 49082508 & 99.376131 \\
\hline
\end{tabular}

Table 4-3 Statistical results of surface water distribution in Guilin in 2016

It can be seen from the regional changes of surface water distribution that the surface water distribution in 2010 is scattered, while the surface water distribution in 2013 and 2016 is concentrated, mostly distributed in the south-central region of Guilin. From the analysis of total surface water, the total surface water is on the rise. The distribution of surface water in the distribution area is small change in the large river basin, small watershed and small tidal flat in the northwest have big change, showing a gradual trend, and tending to the south (Long, 2007).

\subsection{Data Accuracy Verification}

According to the statistical yearbook data released by the bureau of statistics of Guangxi Zhuang Autonomous Region, the surface water resources of Guilin in 2010, 2013 and 2016 are shown in figure 4-2.

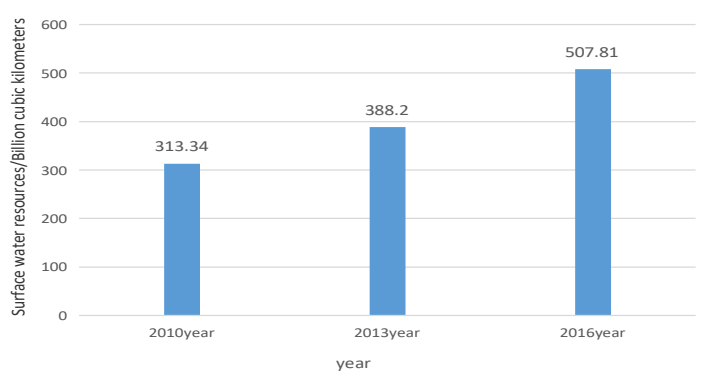

Figure 4-2 surface water resources of Guilin in 2010, 2013 and 2016

Annual runoff of Guijiang river in 2010, 2013 and 2016 in Guilin is shown in figure 4-3.

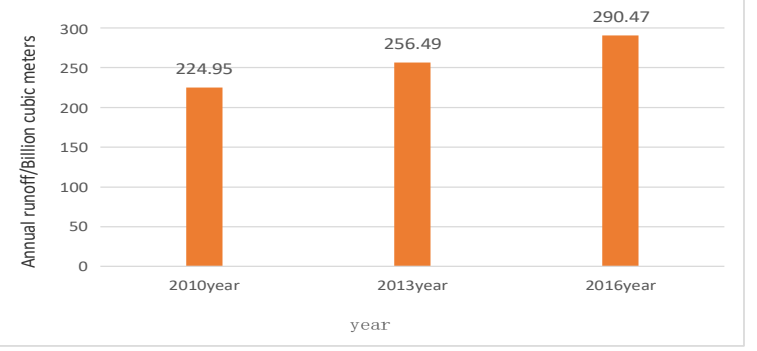


Figure 4-3 annual runoff of Guijiang river in 2010, 2013 and 2016 in Guilin

From the data in Figure 4-2 and Figure 4-3, it can be seen that the amount of water resources in Guilin City has increased from 2010 to 2016, and the annual runoff has also increased year by year, which can verify the 2010 and 2013 years extracted from this study. And the trend of the increasing distribution of surface water in Guilin in 2016 is correct.

\section{INFLUENCE OF NATURAL PROCESSES AND HUMAN ACTIVITIES ON THE SURFACE WATER}

\subsection{Natural Factors}

The temperature of the study area from 2010 to 2016 is generally on the rise, as shown in Figure 4-4.

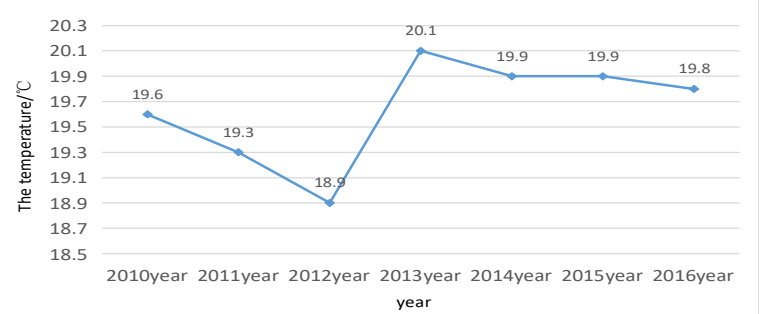

Figure 4-4 annual average temperature changes in Guilin from 2010 to 2016

According to the average temperature value in the table, the average annual temperature in Guilin increased by $0.2{ }^{\circ} \mathrm{C}$ between 2010 and 2016.From 2010 to 2012, the annual average temperature in Guilin declined, and by 2013 it suddenly increased by $1.2{ }^{\circ} \mathrm{C}$. Under the same conditions, the rise of temperature accelerates the evaporation process of the water surface, The rise of temperature has accelerated the evaporation process of the water surface, causing the surface area of the surface water to shrink, The tidal flats are more exposed to the water surface, and the areas of rivers, paddy fields, farms, lakes, reservoirs, etc. are shrinking (Sun et al., 2005). In 2013, the scattered surface water in the northwestern region was significantly reduced compared with that in 2010 . This indicates that the temperature change in the study area coincides with the change of surface water area during the study period, indicating that the change of surface water area has a certain relationship with the temperature change.

Atmospheric precipitation is the main source of recharge water for surface water. The annual precipitation in the study area shows a trend of increasing and decreasing, as shown in Figure4-5.

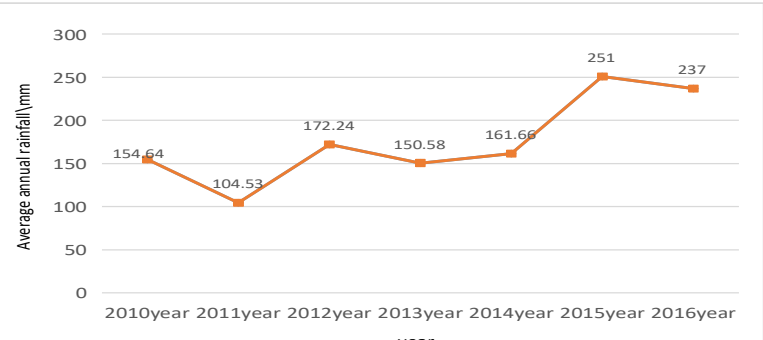

Figure 4-5 changes of annual average rainfall in Guilin from 2010 to 2016
The rainfall in Guilin is increasing from north to south, and the rainfall is most abundant from March to August, but it gradually decreases from September to February. As you can see from Figure 4-5, the rainfall in 2013 is less than in 2010, but the rainfall in 2012 is very rich. Therefore, the reason why there was more water on the surface in 2013 than in 2010 May be caused by the large amount of water storage in 2012 .

The 2016 data is in the month of heavy rainfall, therefore, the amount of surface water in the southern region increased in 2016 compared with that in 2010 and 2013, the change of surface water area in the study area is basically consistent with the change in the study period. This indicates that there is a trend correlation between changes in surface water volume and changes in precipitation.

\subsection{Humanistic Factors}

1)The impact of population growth

The total population and urban population of Guilin in 2010 , 2013 and 2016 are shown in figure 4-6.

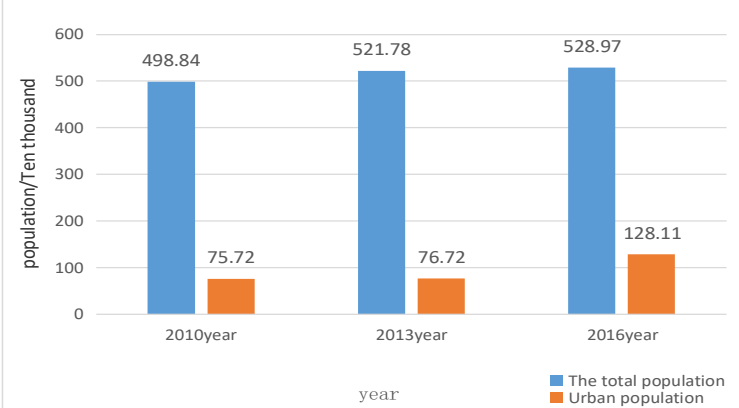

Figure 4-6 total population and urban population of Guilin in 2010, 2013 and 2016

According to figure 4-6, compared with 2010, the population of Guilin increased by about 301,300 in 2016, an increase of $5.70 \%$. Compared with 2010, the urban population in 2016 increased by about 523,900 , an increase of $40.89 \%$.

As the population grows, it is necessary to increase food production. In addition to increasing the output per unit area, the construction of the living environment and infrastructure will inevitably occupy more land, especially paddy fields and tidal flats. From the change of surface water distribution in the study area, the reduction of paddy area and tidal flat area is mainly due to the expansion of the city. At the same time, the development of the garden has increased the area of paddy fields (Gan, et al.,1999). In fact, the paddy field and shoal area in the northwestern part of the study area showed a downward trend, indicating the close relationship between population growth and changes in surface water distribution.

2) Urban development impact

During the three periods of the study, the reduction of surface water area in the northwestern part of the study area was caused by the expansion of urban and rural residential land using tidal flats, paddy fields and urban inland rivers. According to statistics, from 2010 to 2016, the urban area has increased by 47.63 square kilometers, Expanded by $7.77 \%$. In the past six years, the area of paddy fields and tidal flats in the northwest and central part of the study area has been decreasing, and most of them have become urban land (Liu et al., 2001). In addition, due to the influence of the image accuracy of the study area, the 
area of surface water is not completely counted in this study, but it is obvious from the distribution of the image that the surface water distribution changes the position of the area.

3 ) Government decision-making influence

After 2010, Guilin City also formulated a series of preferential policies for tourism, which promoted the vigorous development of tourism and promoted the development of various water upstream play projects. The development of water recreation projects has made water resources more important, and the government has increased the protection of water resources and water storage (Dong et al., 2012).

\subsection{Protection measures}

In the early stage of urban planning, the protection and utilization of surface water resources should be planned from a long-term perspective. In view of Guilin, tourism projects related to water resources can be planned comprehensively. If the water quality is good, the river section with characteristic landscape on both sides can be considered to protect and use its landscape function.

Strengthen water tracking and monitoring. To make prompt judgments, decisions and treatment of changes in water quality and pollution events. Strengthen management and improve management institutions. Establish a unified water resource management and protection institution.

\section{CONCLOSION}

This article uses Guilin as a research area, using Landsat 5 and Landsat 8 data sources to perform optimal band combination on images in ENVI software. Considering that the correlation between the bands is the least for the water body information extraction, the combined band color is as close as possible to the real object, so the R5G4B3 band is selected for combination. The supervised classification method was used to extract water body information, and the remote sensing dynamic change monitoring research was carried out on the surface water distribution in the study area. Combined with the hydrological resource data of Guilin City, the human factors and natural factors affecting the distribution of surface water distribution in the research area were analyzed. The conclusion is as follows: 1. The distribution of surface water in Guilin City is extremely extreme. From the image point of view, the surface water in the northwest has suddenly decreased, while the central and southeastern regions have increased.

2. With the increase of population and the expansion of urban areas, areas such as cultivated land, paddy fields and tidal flats will be occupied. Therefore, urban development is also an important factor affecting surface water changes.

3. The awareness of water resources protection is weak. Large areas of tidal flats and river crossings are encircled into cultivated land, aquaculture areas and industrial development zones, causing irreparable ecological environmental damage and wasteful loss of valuable water resources. It is necessary to strengthen the awareness of water resources protection.

4. The protection and development of water resources in Guilin is closely related to the government's tourism policy.

In this study, only three different time periods of data were used for analysis, the time span was small, and the water body changes were not obvious enough. When analyzing the causes of changes in surface water distribution, only the annual interval change analysis was performed, and the month in which the selected image data was used was not analyzed in detail. In the next study, the time span and time interval can be increased to improve the accuracy of the water body change analysis, and the monthly analysis of the image data can be analyzed in detail to improve the analysis.

\section{REFERENCES}

Zhu, H., 2003. Application research of remote sensing technology in water monitoring of surface water source. China water resources and hydropower research institute,.

Yan Wang. Dynamic remote sensing monitoring and landscape pattern analysis of ruoergai wetland [D]. China university of geosciences (Beijing),2007.

Hui Li, Changan Li,Lihua Zhang,et al.Study on the relationship between lake area and water level of Poyang lake based on MODIS image [J].Quaternary study,2008,28(2):332-337.

Xiwei Fan, Yuanli Xie, Zhijun Li, et al. Monitoring of surface water resource changes in Lanzhou and Xining based on RS [J].groundwater,,2010,32(5):105-107.

Chaoxun Liu. Research on remote sensing simulation and application of regional scale surface water heat -- a case study of Shandong province [D].Nanjing university of information technology,2008.

Yingshi Zhao. Principles and Methods of Remote Sensing Application Analysis [M], Science Press, 2003, 6.

Shafian S,Maas S.Index of Soil Moisture Using Raw Landsat Image Digital Count Data in Texas High Plains[J]. Remote Sensing,2015,7(3):2352-2372.

Yuerong Wang.Study on the Influence of Tourism Development on Landscape Structure of Minjiang River Basin[D].Central South Forestry College,2003.

Guo Chen,Hongfu Zuo. Adaptive fuzzy threshold segmentation of images [J].Journal of automation, 2003, 29 (5).

Wanqi Bai, Shidong Zhao. A Review of Research Models on Land Use and Land Cover Change[J].Journal of Natural Resources, 1997(2):169-175.

Jieyun Long. Application of layered extraction of feature information in remote sensing thematic mapping [D]. Master thesis of Nanjing Normal University, 2007, 5.

Minzhang Sun,Zuoxin Liu,Wu, B. F., 2005.Satellite remote sensing ET method and its application in water management. Advances in water science, 16(3):468-474.

Puping Gan, Runsheng Wang, Yongjiang Wang,et al.Classification method of land use and land cover based on remote sensing technology [J].Remote sensing of land and resources, 1999(4):40-45.

Yujie Liu, Zhongdong Yang.Remote sensing information processing principle and algorithm of MODIS [M].Beijing: science press, 2001

Linqin Dong, Guangxin Zhang,Guoqing Wang,et al. Analysis of the contribution of natural variation of climate to the assessment of the impact of climate change on water resources. Model and method of base period[J]. Advances in water science,,2012,23(2):147-155. 